\title{
Supporting Information for Quantifying the Surface Coverage of Conjugate Molecules on Functionalized Nanoparticles
}

Leonard F. Pease III, ${ }^{1}$ De-Hao Tsai, ${ }^{1,2}$ Rebecca A. Zangmeister, ${ }^{1}$ Michael

R. Zachariah, ${ }^{* 1,2}$ Michael J. Tarlov*, ${ }^{1}$

1. National Institute of Standards and Technology (NIST), 100 Bureau Drive MS

8362, Gaithersburg, MD 20899

2. The University of Maryland, 2181 Glenn L. Martin Hall, College Park, MD

20742

*Email: mtarlov@nist.gov,mrz@umd.edu

Material and Preparation: Commercially available monodisperse Au colloids (nominally $20 \mathrm{~nm}, 7 \times 10^{11}$ particles/mL, citrate stabilized, Ted Pella Inc.) were employed herein. Commercial custom oligonucleotides were synthesized and HPLC-purified by the vendor and used as received without further purification. The 5' thiol-modified oligonucleotides were used without removing the protective $\mathrm{S}-\left(\mathrm{CH}_{2}\right)_{6} \mathrm{OH}$ group from the $5^{\prime}$ end as described in Petrovykh, D. Y., et al. ${ }^{1}$ For brevity, these oligonucleotides are referred to as $\mathrm{T}_{\mathrm{x}} \mathrm{SH}$ where $x$ represents the number of thymine bases per strand, between 5 and 30 .

Conjugated Au colloids were prepared by adding DNA at $200 \mu \mathrm{mol} / \mathrm{L}$ to the particles in the ratio of $50 \mu \mathrm{L}$ per $850 \mu \mathrm{L}$ of gold nanoparticles and the solution was allowed to react $\geq 18 \mathrm{~h}$. Salts were added in two stages. First, $20 \mu \mathrm{L}$ of $5 \mathrm{~mol} / \mathrm{L} \mathrm{NaCl}$ and $10 \mu \mathrm{L}$ of $1 \mathrm{~mol} / \mathrm{L} \mathrm{K}_{2} \mathrm{HPO}_{4}$ were added per $850 \mu \mathrm{L}$ of particles. Second, after $\geq 3$ hours an additional $149 \mu \mathrm{L}, 373 \mu \mathrm{L}$, or 746 $\mu \mathrm{L}$ was added to achieve the desired ionic strength, after which the reaction proceeded for $\geq 48$ h. To determine whether 48 hours was sufficient to reach a saturated steady state, we allowed a solution of $\mathrm{T}_{30} \mathrm{SH}$ to react for 6 weeks and found no statistical difference in the size from the sample reacted for 48 hours. This result is commensurate with the work of Steel et $\mathrm{al}^{2}$, who find the surface coverage to be greater than $90 \%$ complete within 5 hours-substantially less than the 48 hours here. Thus, the surface coverage may be expected to be fully saturated.

The Au colloid solution was then centrifuged (Model 5415D with aerosol-tight, 24 place, fixed $45^{\circ}$ rotor, Eppendorf, Inc.) at $1380 \mathrm{rad} / \mathrm{s}(13200 \mathrm{rpm})$ with radius of $11 \mathrm{~mm}$ for $10 \mathrm{~min}$ in order to separate the colloids from the supernatant and to reduce the nonvolatile salt concentration. The supernatant was removed, leaving less than $20 \mu \mathrm{L}$ in the vial, and replaced with an equivalent volume of $>18.0 \mathrm{M} \Omega \cdot \mathrm{cm}$ water. The Au colloids easily redispersed by vortex mixing. Centrifugation followed by replacement of the supernatant with water more than one time resulted in flocculation of the bare Au colloids. In contrast, colloids coated with DNA to which subsequent aliquots of salt solutions were added remained stable through duplicate centrifugation runs.

Following centrifugation, typically $60 \mu \mathrm{L}$ of a $20 \mathrm{mM}$ ammonium acetate buffer (Sigma, $99.9 \%, 0.2 \mathrm{~S} / \mathrm{m}, \mathrm{pH} 8$ ) was added to $900 \mu \mathrm{L}$ of solution to provide the requisite conductivity for the electrospray system. The addition proceeded in $10 \mu \mathrm{L}$ aliquots interspersed by vortex mixing for $\geq 5$ seconds, since direct addition tended to induce particle flocculation particularly for the bare particles. 
The experimental system consists of an electrospray aerosol generator (Model 3480, TSI Inc.), a modified differential mobility analyzer (Model 3080n, TSI Inc.) and a condensation particle counter (Model 3025, TSI Inc.). A high flow rate mass flow controller allows for flow rates in excess of those originally specified by the manufacturer but still within the laminar flow regime. We compared the sizes measured at $30 \mathrm{~L} / \mathrm{min}$ with those at $10 \mathrm{~L} / \mathrm{min}$ to find similar sizes but with greater resolution. First, the electrospray aerosol generator transferred the immersed particles into small droplets. A vial containing the Au colloids ( $>100 \mu \mathrm{L}$ ) was placed under pressure, which drove the sample solution to the nozzle through a capillary $(25 \mu \mathrm{m}$ in diameter, $\approx 24 \mathrm{~cm}$ in length) at approximately $66 \mathrm{~nL} / \mathrm{min}$.

The shape of the meniscus at the tip of the capillary controls the droplet size distribution. The shape is a function of both the sheath gas flow rate, which we fix at $1.2 \mathrm{~L} / \mathrm{min}(1.0 \mathrm{~L} / \mathrm{min}$ of Air and $0.2 \mathrm{~L} / \mathrm{min}$ of $\mathrm{CO}_{2}$ ), and the electrospray voltage, which we vary typically between $2 \mathrm{kV}$ and $3 \mathrm{kV}$. Proper tuning of the applied voltage yields a stable cone jet flow regime, characterized by a sharp Taylor cone. Achieving this condition is critical because other meniscus regimes at higher or lower voltages failed to produce the narrow distribution of small droplet sizes necessary to achieve high quality, reproducible results. Figure A below characterizes the conditions under which the stable cone jet regime may be obtained as a function of electrospray voltage and ammonium acetate concentration. As the droplets leave the electrospray nozzle they are bombarded by charged ions which effectively reduce or "neutralize" the particles. While some droplets become negative or neutral, those analyzed in the differential mobility analyzer typically possess a single positive charge. Subsequently, the droplets dry leaving particles with the charge acquired during neutralization.

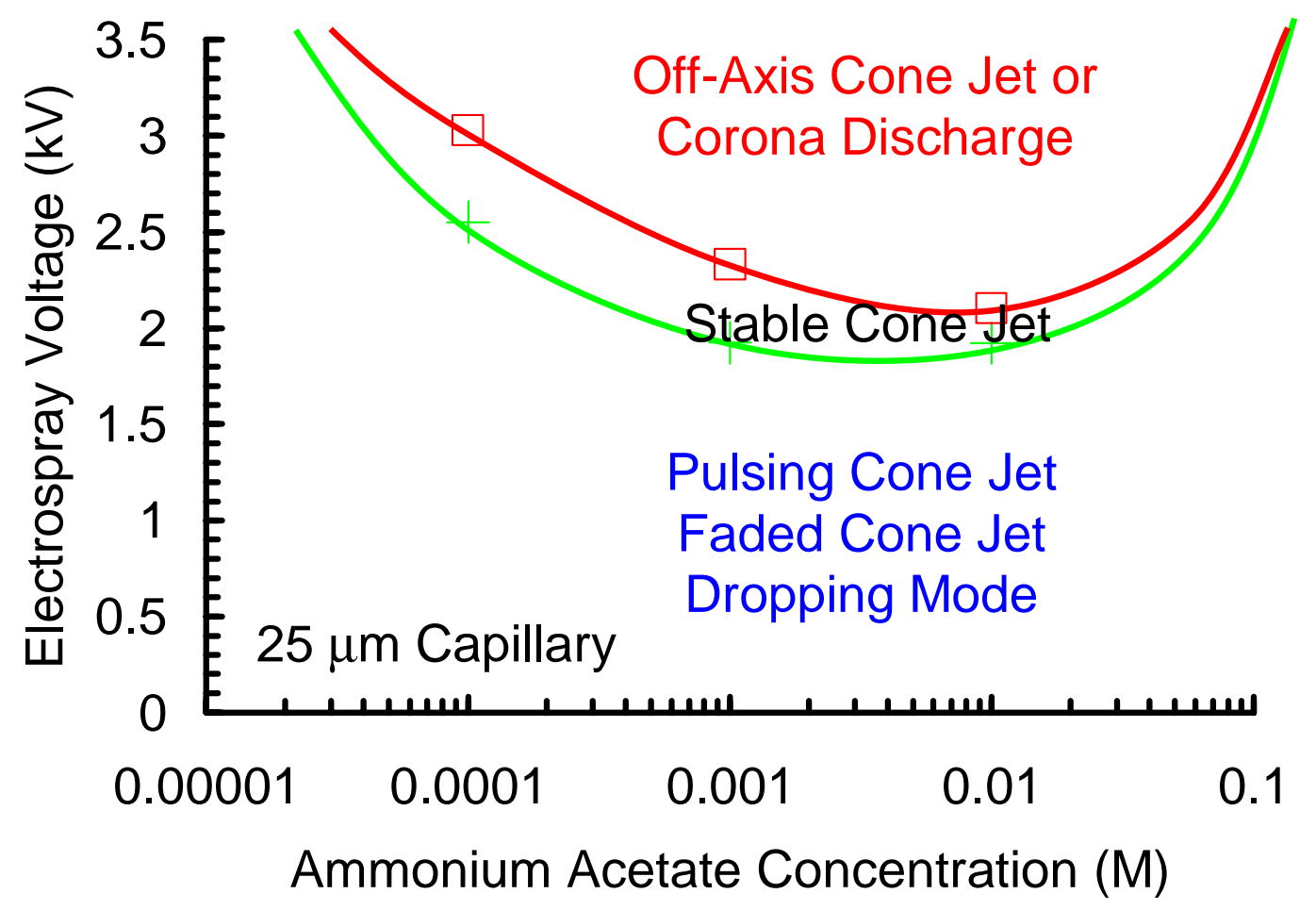

Figure A. Electrospray voltage versus ammonium acetate concentration depicting the conditions within which a stable cone jet flow regime may be obtained. The upper and lower curves separate the stable cone jet regime from the corona discharge and pulsed cone jet, respectively. 
Then, the particles enter the differential mobility analyzer (DMA) to be separated based on their charge-to-drag ratio. Within the DMA a carrier flow of $30 \mathrm{~L} / \mathrm{min}$ of gaseous $\mathrm{N}_{2}$ sweeps particles towards the exit. Meanwhile, the electric field produced by an electrode in the center of the chamber at high voltage (from $0 \mathrm{kV}$ to $-10 \mathrm{kV}$ ) attracts positively charged particles. Particles, for which the electrically attractive force balances the drag force from the carrier flow, enter a collection slit in the central electrode near the exit of the chamber. Since the charge on the particle is known, particles passing through the collection slit into the particle counter are characterized by their mobility diameter. Coated particles display a larger mobility diameter due to their large projected area. At each voltage particles are counted for 20 seconds. Stepping through the voltages yields a mobility size distribution in which a point was collected every 0.2 nm.

To determine the peak location, the mode or maximum of the particle size distribution was selected. Use of other statistics such as the number-average diameter did not significantly improve the results for these particular conditions. We assume droplets emerging from the electrospray to have a uniform size regardless of whether a gold particle is contained therein or not. As the droplet dries, nearly the same volume of salt would be located in each type.

Correcting for the crust of salt with the Kaufman correction assumes the volume of the gold particle $(\approx 20 \mathrm{~nm})$ to be much less than the diameter of the droplet, which we have calculated to be $150-300 \mathrm{~nm}$.

The relationship between the size reported by the DMA and the actual geometry of the particle may be derived from the work of Song, et al. ${ }^{3}$ The drag force exerted on a particle of Stokesian diameter $d$ by a gas with viscosity $\mu$ in the laminar flow regime is $F_{D}=3 \pi \mu \chi d / C_{c}$, where $\chi$, the shape factor and $C_{c}$ is the Cunningham slip correction factor. Our homemade software uses $\mu=1.81 \cdot 10^{-5} \mathrm{~Pa}$ s- the viscosity of air- $C_{c}=1+K n[\alpha+\beta \operatorname{Exp}(-\gamma / K n)]$, where $K n$ $=2 \lambda / d, \alpha=1.257, \beta=0.40, \gamma=1.110$, and the gas mean free path at room temperature $\lambda=66$ $\mathrm{nm}$. The size reported by the DMA is $\chi_{\text {total }} D$ and $\chi_{\text {total }}(D+H)$ and for bare and coated particles respectively, accordingly the remainder of this paragraph focuses on estimating the shape factor. To determine each shape factor in each orthogonal direction, we employ the "equivalent circle" approximation, $\chi_{i} d=\left(4 A_{i} / \pi\right)^{1 / 2}$. This approximation relates the shape factor to the 2-D projection of the area, $A_{i}$, as is appropriate for flows in the free molecular regime. ${ }^{4}$ We note that this approximation only applies when the deviation of any cross section of the particle does not deviate too severely from a circle and is distinct from the "equivalent sphere" approximation that leads to the Russell estimate of the hydrodynamic radius. " Because Brownian motion rotates the particle through all possible orientations on average, Song, et al., show the inverse of the total dynamic shape factor, $\chi_{\text {total }}$, to be the sum of the inverses of the individual shape factors, $\chi_{i}$, in each orthogonal direction or $1 / \chi_{\text {total }}=\left(1 / \chi_{1}+1 / \chi_{2}+1 / \chi_{3}\right) / 3$. Multiplying through by the diameter and employing the equivalent circle approximation then gives $D+H=\left(\pi^{1 / 2} \Sigma_{i} A_{i}^{-1 / 2} / 6\right)^{-1}$.

With this relationship between the coating thickness and the projected area, we now derive the surface coverage for long and flexible strands from the coating thickness. We employ a "lumpy sphere" model approximating each strand as a hemispherical cap. The projected area for the particle and the adornment is $A_{i}=\pi D^{2} / 4+N_{m} \pi\left\langle x^{2}\right\rangle / 2$ in each direction, where $N_{m}$ represents the number of hemispherical caps of radius $\left\langle x^{2}\right\rangle^{1 / 2}$ around the circumference. Knowing the brush thickness then allows us to calculate $N_{m}$ for a given value of the Kuhn length. Since $N_{m}$ represents the number of caps around the circumference of the particle, $N_{m} / \pi D$ represents their linear density. Squaring this then gives $\sigma=\left(N_{m} / \pi D\right)^{2}$, which represents the area density or surface density of the caps. Putting it all together yields $\sigma=\left[(D+H)^{2}-D^{2}\right]^{2} /\left[2 \pi D<x^{2}>\right]^{2}$ or 
$\sigma=\left[(D+H)^{2}-D^{2}\right]^{2} /\left[\pi D c^{2} N_{b} N_{k} l_{b}^{2}\right]^{2}$, which relates the coating thickness, the diameter of the particle, the number of bases, the number of bases per Kuhn length and the length of a base to the surface coverage (see Table A).

The validity of this analytical model may be determined by comparing the results of this equation with similar computational results for a "lumpy sphere" at the close packed condition." For close packed hemispherical caps of radius $a$ on a circumference, $N_{m}=\pi R / a$ and $A_{i}=\pi R^{2}(1+\pi a / 2 R)$. The radius of the adorned particle is then $R_{\text {lumpy }}=R(1+\pi a / 2 R)$. Substitution of $a / R=1 / 10,1 / 15$, and $1 / 20$ finds $R_{\text {lumpy }} / R=1.07568,1.05106$, and 1.03853 , respectively. By way of comparison, Mansfield, et al., compute the Stokesian radius for lumpy spheres, in their notation $C$, as $C / R=1.07285,1.04974$, and 1.03704, respectively. The agreement between our analytical model and their computations is better than $0.3 \%$ validating our model for this specific case.

Table A. Summary of Raw Data

\begin{tabular}{|c|c|c|c|c|c|c|c|c|}
\hline & $N_{b}$ & $\begin{array}{c}\text { Added Salt } \\
\text { Concentration } \\
(\mathrm{mol} / \mathrm{L})\end{array}$ & $\begin{array}{c}\text { Salt Peak } \\
(\mathrm{nm})\end{array}$ & $\begin{array}{c}\text { Au+DNA } \\
\text { w/Salt Crust } \\
(n m) \\
\end{array}$ & $\begin{array}{c}\text { Au+DNA } \\
\text { w/o Salt } \\
\text { Crust (nm) } \\
\end{array}$ & $\begin{array}{c}<x^{2}>^{1 / 2} \\
(\mathrm{~nm})\end{array}$ & $\begin{array}{c}\sigma \text { w/Primary } \\
\text { Method }\left(10^{13}\right. \\
\left.\text { molecules } / \mathrm{cm}^{2}\right)\end{array}$ & $\begin{array}{c}\sigma \text { w/Alternate } \\
\text { Method }\left(10^{13}\right. \\
\left.\text { molecules } / \mathrm{cm}^{2}\right) \\
\end{array}$ \\
\hline$\overline{\text { bare }}$ & 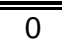 & $\overline{00.00}$ & 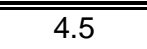 & 21.0 & 20.9 & - & - & - \\
\hline$(\mathrm{dT})_{5} \mathrm{SH}$ & 5 & 0.13 & 9.2 & 23.2 & 22.7 & 1.42 & 3.63 & 3.34 \\
\hline$(\mathrm{dT})_{5} \mathrm{SH}$ & 5 & 0.13 & 9.0 & 23.0 & 22.5 & 1.42 & 2.93 & 2.99 \\
\hline$(\mathrm{dT})_{10} \mathrm{SH}$ & 10 & 0.13 & 10.0 & 23.2 & 22.6 & 2.00 & 0.76 & 1.59 \\
\hline$(\mathrm{dT})_{15} \mathrm{SH}$ & 15 & 0.13 & 9.0 & 23.4 & 22.9 & 2.45 & 0.52 & 1.35 \\
\hline$(\mathrm{dT})_{20} \mathrm{SH}$ & 20 & 0.13 & 10.6 & 23.8 & 23.1 & 2.83 & 0.33 & 1.09 \\
\hline$(\mathrm{dT})_{30} \mathrm{SH}$ & 30 & 0.13 & 10.0 & 24.0 & 23.4 & 3.47 & 0.20 & 0.86 \\
\hline$(\mathrm{dT})_{5} \mathrm{SH}$ & 5 & 0.80 & 6.0 & 23.4 & 23.3 & 1.42 & 6.36 & 4.48 \\
\hline$(\mathrm{dT})_{10} \mathrm{SH}$ & 10 & 0.80 & 5.4 & 24.2 & 24.1 & 2.00 & 3.03 & 3.29 \\
\hline$(\mathrm{dT})_{15} \mathrm{SH}$ & 15 & 0.80 & 4.6 & 25.2 & 25.1 & 2.45 & 2.46 & 3.09 \\
\hline$(\mathrm{dT})_{20} \mathrm{SH}$ & 20 & 0.80 & 5.8 & 26.0 & 25.9 & 2.83 & 1.98 & 2.84 \\
\hline$(\mathrm{dT})_{20} \mathrm{SH}$ & 20 & 0.80 & 4.8 & 25.4 & 25.3 & 2.83 & 1.53 & 2.46 \\
\hline$(\mathrm{dT})_{5} \mathrm{SH}$ & 5 & 1.52 & 7.6 & 23.8 & 23.5 & 1.42 & 7.99 & 5.06 \\
\hline$(\mathrm{dT})_{10} \mathrm{SH}$ & 10 & 1.52 & 7.4 & 25.2 & 25.0 & 2.00 & 5.09 & 4.36 \\
\hline$(\mathrm{dT})_{15} \mathrm{SH}$ & 15 & 1.52 & 5.8 & 26.2 & 26.1 & 2.45 & 3.85 & 3.94 \\
\hline$(\mathrm{dT})_{20} \mathrm{SH}$ & 20 & 1.52 & 6.6 & 27.2 & 27.1 & 2.83 & 3.17 & 3.68 \\
\hline$(\mathrm{dT})_{20} \mathrm{SH}$ & 20 & 0.93 & 11.6 & 26.2 & 25.4 & 2.83 & 1.59 & 2.51 \\
\hline$(\mathrm{dT})_{5} \mathrm{SH}$ & 5 & 2.30 & 7.8 & 24.2 & 23.9 & 1.42 & 10.69 & 5.90 \\
\hline$(\mathrm{dT})_{10} \mathrm{SH}$ & 10 & 2.30 & 9.4 & 26.0 & 25.6 & 2.00 & 6.86 & 5.13 \\
\hline$(\mathrm{dT})_{15} \mathrm{SH}$ & 15 & 2.30 & 8.2 & 27.2 & 26.9 & 2.45 & 5.38 & 4.75 \\
\hline$(\mathrm{dT})_{20} \mathrm{SH}$ & 20 & 2.30 & 8.4 & 28.0 & 27.7 & 2.83 & 4.01 & 4.21 \\
\hline
\end{tabular}

Alternate method assumes $\rho_{a v}=0.89 \mathrm{~g} / \mathrm{cm}^{3}$ for $(\mathrm{dT})_{25} \mathrm{SH}$ at $1 \mathrm{~mol} / \mathrm{L}$ of $\mathrm{NaCl}$ added. ${ }^{1}$

FTIR Analysis: Conjugated Au colloids were concentrated by centrifugation and then dropped onto commercially available Teflon IR cards. Ethanol was used to aid in wetting the Teflon surface with the aqueous Au colloid solution that was then allowed to dry in air. Transmission FTIR absorption spectra were measured using a commercially available spectrometer with a cryogenic mercury cadmium telluride detector in transmission mode. Presented spectra result from averaging 128 scans at $4 \mathrm{~cm}^{-1}$ resolution. All FTIR measurements were performed on freshly prepared samples. The peak at $\sim 1697 \mathrm{~cm}^{-1}$ is assigned to free carbonyl groups found in the thymine bases; the peak at $\sim 1574 \mathrm{~cm}^{-1}$ is assigned to carbonyl groups of the thymine bases 
that are chemisorbed to the gold surface. The relative intensity ratio of the peak at $1697 \mathrm{~cm}^{-1}$ to the peak at $1574 \mathrm{~cm}^{-1}$ increases at higher salt concentration suggesting that the number of thymine bases raised from the surface of the particle is greater at the higher salt concentration. This is consistent with mildly overlapping islands.

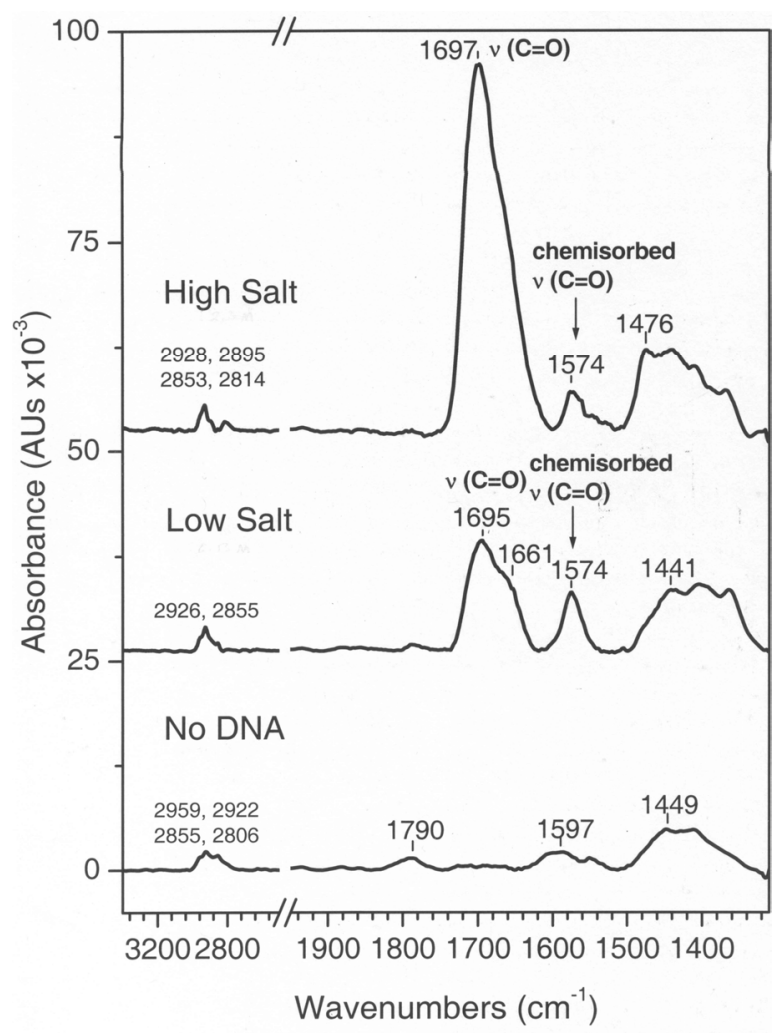

Figure B. Infrared spectra of bare gold particles, gold particles with ssDNA prepared with 0.13 mol/L salt (i.e. low salt), and gold particles with ssDNA prepared with $2.3 \mathrm{~mol} / \mathrm{L}$ salt (high salt).

Disclaimer: The reference to commercial equipment does not imply its endorsement by the National Institute of Standards and Technology (NIST).

\section{References:}

(1) Petrovykh, D. Y.; Perez-Dieste, V.; Opdahl, A.; Kimura-Suda, H.; Sullivan, J. M.; Tarlov, M. J.; Himpsel, F. J.; Whitman, L. J. Journal of the American Chemical Society 2006, $128,2$.

(2) Steel, A. B.; Herne, T. M.; Tarlov, M. J. Analytical Chemistry 1998, 70, 4670.

(3) Song, D. K.; Lenggoro, I. W.; Hayashi, Y.; Okuyama, M.; Kim, S. S. Langmuir 2005, 21, 10375 .

(4) Friedlander, S. K. Smoke, Dust, and Haze, Second ed.; Oxford University Press: New York, 2000.

(5) Mansfield, M. L.; Douglas, J. F.; Irfan, S.; Kang, E. H. Macromolecules 2007, 40, 2575. 